\title{
The Research Progress of Transforming Growth Factor (TGF - Beta) Gene Regulation and Invasion and Metastasis of Pancreatic Cancer
}

\author{
Xiao-jing Gong (review), Guiqing Yang, Dongning Sun, Lingqin Wan, Yi-shan Wang (review) \\ Binzhou Medical University affiliated yantai city's people's liberation army 107 hospital treatment and noninvasive \\ diagnostic center, yantai 264002
}

\begin{abstract}
Transforming growth factors TGF is a kind of a superfamily of peptides, which have strong sensitivity to cell proliferation、 differention and transfer, its function and its structure are very complicated, and it is a cell factor which widely exist in multiple cells. There are more and more attention on it in the recent years, especially the relation with the tumor is become a key point. TGF-ßcan inhibit related proteins and gene regulation at the early stages of the tumor ,so can effect the cell proliferation, but at the late stages the tumor, TGF- $\beta$ can also promote the invasion and matastasis of tumor cells. The important feature of pancreatic cancer is a highly mortality rate. But TGF- $\beta$ have two-direction adjust effect to tumor. This article is mainly to the TGF - beta gene expression and the mechanisms and the invasion and metastasis of pancreatic cancer are reviewed in this paper.
\end{abstract}

Keywords-transforming growth factors- $\beta$; gene expression; pancreatic cancer; invasion and metastasis

Pancreatic cancer has a very level of deterioration, and the diagnosis of early and late treatment for pancreatic cancer has certain difficulties, is the clinical common and difficult to cure tumor. Its morbidity and mortality, both at home and abroad in recent years has significantly increased, at the same time also is one of the worst prognosis of malignant tumors, and finally able to in the clinical diagnosis of late, mostly are so pancreatic cancer treatment effect is not very good. The 5-year survival rate is only $4 \% \sim 5 \%$. And it is one of the main biological behavior is highly invasive and metastatic [1].According to consult literature material, TGF - beta can affect a variety of cell growth, proliferation and differentiation, and TGF - beta also have inhibition to pancreatic cancer cells and promote growth of two-way effect [2]. Hence TGF - beta in malignant tumors, especially in the expression of pancreatic cancer will become the anti-tumor treatment, a new study.

\section{TGF - BETA GENE EXPRESSION REGULATION}

\section{A. TGF- beta subtype, structure and distribution}

TGF supergene family has many kinds, and TGF beta is one of the important members, TGF - beta is a dimer proteins, peptides, mainly have two as, and more than 40 members have been determined..TGF - beta highly conserved in evolution. TGF - beta 5 kinds of isomer: TGF beta 1 , TGF beta 2 , TGF beta 3 , TGF beta 4 , 5 , TGF beta before three receptor exists in the mammalian body, at the same time TGF beta 1, TGF beta 2, TGF beta 3 among these three receptor precursor molecules contain some conservative area, there are 7 exons, and biological functions are similar, and amino acid sequence homology, containing propeptide of inactive TGF - beta precursor compounds form synthesis, generally referred to as the precursor of TGF - beta (catant TGF - beta) [3].TGF beta $1 \sim$ TGF - although beta 3 structure similar, but different biological activities [4]. The function of each isomer in the body is a specific and concrete, and the cross function there will be no interference with each other, and transforming growth factor superfamily member and TGF - beta 1 has a similar sequence, the carboxyl end and at the same time is known as the precursor molecules, and again in the carboxyl end have a common characteristic, contains nine conservative cysteine residues. Previous experiments, TGF - beta are mainly distributed in the bones and platelet [5].

\section{B. TGF-beta receptors and signal transduction pathways}

1) TGF-beta receptors. There are three kinds of TGF beta receptors: TGF - beta receptors I I beta $\mathrm{R}(\mathrm{T})$, TGF - beta receptors II II beta R (T), TGF - beta receptors III III beta $\mathrm{R}(\mathrm{T})$. TGF - almost beta receptors exist with all the normal cells and cultured tumor cell membrane surface, this three kinds of receptors with high affinity membrane surface binding protein. And TGF beta is mainly by T R I beta and T beta R II play a role of biology. But $\mathrm{T}$ beta $\mathrm{R} \mathrm{I}$ and $\mathrm{T}$ beta $\mathrm{R}$ II receptor on the amino acid sequence and structure differences. The I receptor for 500 amino acids, II receptor for 570 amino acids [6]. When I receptor is activated, it can separate signal transduction, its characteristic is the key to it is "SGSGSG" sequence, and the sequence is a key link in the process of receptor kinase activity can have [7], and II receptor is a subordinate receptor beta polysaccharide and endothelial glycoprotein, is a major receptor of fibroblasts and epithelial cells, it can itself phosphorylation, and TGF - beta directly, giving play to the role of signal transduction. 
2) TGF - beta signal transduction pathways - Smads protein. TGF - beta signal transduction path signal molecule has a lot of, but by with smads cytoplasm protein is given priority to, by a lot of attention in recent years. TGF - beta is mainly by T R I beta and T beta R II receptor activation smad pathway, smads protein is composed of three parts: the amino end, carboxyl end MH1 and MH2 structure domain and spacer [8].Previous experiments have confirmed that the Smads protein there are three main types: receptor activation sex type (receptor - activated smad, R - smad) : smad1, smad2 and smad3, smad5, smad8, this category can be $\mathrm{T}$ beta $\mathrm{R} I$ receptor activation and make its phosphorylation, and then combined with Co - Smads and transferred to the cells and specific signal transduction. A longitudinal joint channel type (common - parter smad, Co - smad) : smad4, a subtype of mainly exist in mammals, is also one form only, is different from TGF - beta induce gene expression regulation and all TGF - beta key transcription factor signal must be transferred to a nucleus. $f$ inhibitory smad (inhibitory smad, I - smad) : smad6, smad7, both subtype can I works directly with the TGF - beta receptors, inhibit and adjust the signal transduction of TGF - beta family, the same as the samd4, excluding SSXS structure their carboxyl end, at the same time the TGF - beta signal transduction pathways play a negative regulation role [9].

\section{REGULATING THE EXPRESSION OF TGF - BETA IN PANCREATIC CANCER}

\section{A. Early pancreatic cancer tumor inhibition}

TGF - beta tumor inhibition is embodied in many aspects, it has a very wide range of effects at the same time, for many types of cancer cells play an important role. TGF - beta by smad pathway and the smad pathway of apoptosis regulation, at the same time through the TGF - beta/smad inhibition of cell cycle and paracrine way to control the growth of cells. In the early pancreatic cancer mainly rely on a silk/threonine phosphatase on [10].TGF $\mathrm{c}$ - beta is mainly to tumor suppressor genes affecting cell proliferation inhibition of myc. At the same time also can inhibit cell cycle protein (cyclins) to lower the activity of CDK2 and CDK4, suppressing pancreatic cancer cells from $\mathrm{G} 1$ phase to $\mathrm{S}$ phase. One of the most important thing TGF - beta can also inhibit ErK activity and its nucleus shift [11].

\section{B. TGF - beta in advanced late pancreatic cancer tumor}

TGF - beta function complex in pancreatic cancer, early and late in tumor cells play a different role, respectively, in the early showed inhibitory effect, but with the development of tumor cells, tumor cells growth inhibition of the TGF - beta is not sensitive, and can promote the growth of tumor cell metastasis. These shifts may be different effect by different pathways associated molecules.
1) TGF - beta immune inhibition of tumor. TGF - beta as in promoting the main immunosuppressive molecules in the process of tumor metastasis, the very important role in the formation and development of tumor, TGF - beta mainly through paracrine role in inhibiting the tumor host immune defense. TGF - beta can inhibit the proliferation of T lymphocytes and cytotoxic effect of activation, thus inhibiting excess peroxide and nitric oxide (NO), so the TGF - beta has a microenvironment of tumor cells themselves and surrounding the dual change [12].In the process of development of pancreatic cancer, before the experiment to confirm the TGF - beta expression in tumor cells will be significantly enhanced, while the corresponding to the inhibition of tumor cell gene PTEN will be significantly reduced. This shows that both in the process of development with the incidence of pancreatic cancer has a certain relevance [13].

2) TGF - beta inducing the formation of tumor blood vessels. TGF - beta are important mediators of blood vessel formation, can be directly or indirectly induce the expression of critical angiogenesis factor, at the same time, the tumor cell survival, growth and metastasis mainly depends on the integrity of the vascular system, previous studies have confirmed that TGF - beta on the reshaping of the blood vessels to function and has key effect to ensure the integrity of the vascular system [14].TGF - beta blood vessels can be induced to grow at the same time, and provides favorable for the growth of micro environment, to promote the formation of blood vessels. Especially in endothelial cells, the TGF - beta functions to regulate the more obvious, and increase the gene expression of endothelial cells, thus activating SMAD1 / SMAD5 / SMAD8, thereby promoting the formation of blood vessels. Research has confirmed that the GF - beta can control inhibitors of angiogenesis induced vascular statin (Angiostatin) and the formation of blood vessels [15].

3) TGF - beta inducing the conversion of cell. Transforming growth factor TGF (transforming growth factor, TGF) beta can promote cell differentiation as well as to inhibit the action of the differentiation. In the process of TGF - beta inducing EMT, epithelial cells gradually lose their original characteristics and transformation, between qualitative characteristics to amplify the power of invasion and metastasis of tumor cells. Past a lot of studies have shown that EMT in tumor metastasis of early in the process of play an important role in regulating [16].At the same time existing studies confirm volker [17] showed that the Ras signaling pathways involved in TGF - such as beta mediated transformation process in epithelial to mesenchymal cells. In pancreatic cancer, TGF - beta can induce tumor cells EMT this process, so that the cells of invasive ability enhancement. 


\section{THE MECHANISM OF INVASION AND METASTASIS OF PANCREATIC CANCER AND RELATED SIGNALING PATHWAY}

\section{A. Pancreatic cancer related regulatory factors and molecular}

Pancreatic cancer related regulatory factors and molecular mechanisms of invasion and metastasis Previous studies have confirmed that in pancreatic cancer and the deterioration of the late transfer will appear in the process of the regulation of many factors, and these factors will because cancer cells in the corresponding changes. Further understanding of the related regulatory factors and their development is an important link in advanced pancreatic cancer can early diagnosis and treatment. [18].

1) Vascular endothelial growth factor(VEGF).Vascular endothelial growth factor(VEGF) Previous studies confirmed that VEGF are highly expressed in a wide variety of tumor cells, and to some extent has inhibitory effect on tumor cells. And for the growth of pancreatic cancer metastasis and prognosis also plays a role, is the best known active degree, exclusive of the highest regulatory factors. And the content of VEGF in pancreatic cancer tissue than in normal pancreatic tissue increased obviously [19]

2) Thrombospondin-1(TSP). Platelet response element (TSPs) is mainly in the blood vessels in the operation of the line to play a key role in negative control, especially more obvious effect on the generation of blood vessels in tumor cells. Although the role of the TSP - 1 so important, but the TSP - 1 adjustment mechanism of the formation of new blood vessels is not very clear. But Qian [20] such as found in $85 \%$ of TSP - 1 is highly expressed in pancreatic cancer, but not seen in normal pancreatic tissue abnormalities. But most have proved that the TSP - 1 will appear before the tumor invasion [21].

3) Chemokines and invasion and metastasis of pancreatic cancer. Early in the research of chemokines, especially in the role of pancreatic cancer, think chemokine plays an negative regulatory role in pancreatic cancer. But in recent years, a growing number of studies have demonstrated that in the invasion and metastasis of pancreatic cancer chemokines play a key role, it can induce the formation of new blood vessels, provides the conditions for the transfer of tumor cells, but also can enhance the invasive ability of cancer cells [22].Chemokines are mainly through its receptor CXCR4 constitute the SDF - 11 CXCR4 axis to regulate the invasion and metastasis of tumor cells.

\section{B. Invasion and metastasis of pancreatic cancer}

In the invasion and metastasis of pancreatic cancer related signal transduction pathways. At various stages of the development of pancreatic cancer have different signal transduction pathways activated and play an important role in the invasion and metastasis. And the related signal pathways in pancreatic cancer will change in the process, they are independent and interrelated with each other, make the growth of tumor cells have stronger invasive ability [23].Now with the growth of the signaling pathway and mitotic make concentrated signaling pathways, including can provide powerful basis for symptomatic combination in the future.

1) Growth factor signaling pathway. In the process of invasion and metastasis of pancreatic cancer will appear a lot of related growth factor receptors, many studies have shown that TGF - beta signaling pathways in pancreatic cancer has an important regulating role in the growth and differentiation. The invasion and metastasis of pancreatic cancer has many key factor receptors, by to the epidermal growth factor receptor (EGFR) signaling pathway. EGFR as an important channel of invasion and metastasis of pancreatic cancer, it can activate a variety of cancer cell signaling pathways, at the same time cut expression of EGFR in pancreatic cancer is closely related to the growth and metastasis of pancreatic cancer, at the same time also has an important influence on the prognosis of tumor cells [24].

2) Mitosis signaling pathways Mitosis. Mitosis signaling pathways Mitosis signaling pathway plays a key role in developing pancreatic cancer, there are a number of studies have proved that $\mathrm{P} 13 \mathrm{~K}$ signaling pathways (phosphoinositide13 - kinase) almost there with all the tumor cell signaling pathways, as this is especially true for pancreatic cancer, P13K can be RAS activation, many of them can adjust the size of the cells, proliferation and survival [25].P13K signaling pathway on the growth of pancreatic cancer cells transfer research more and more attention, at the same time also have confirmed institute $\mathrm{P} 13 \mathrm{~K}$ in play a role of anti-tumor plays an important role. And if, after remove the RAS protein expression P13K signaling pathway activation of the transplanted tumor and can keep its original malignant phenotype [26].

IV. TGF - BETA TARGETED ANTI-TUMOR DRUGS IN Clinical ApPlication TGF - BETA Has A SPECIAL BidiRECTIONAL REgUlation ON TUMOR CELls, Especially In The Middle-Late Stage Of The Tumor And The TgF - Beta Will Promote The Growth OF Tumor Cell Metastasis. This MaKes The TgF RESISTANT BETA RESEARCh BECOME A NEW BRIGHT SPOT

\section{A. TGF - beta receptor kinase inhibitor application Previous}

TGF - beta receptor kinase inhibitor application Previous studies have proved that on type I receptor kinase activity can be classic and the classic downstream signaling pathways. Kinase inhibitors main effect on the ATP binding site, at the same time TGF - beta kinase inhibitors are gradually into clinical trials, including ATP binding site (scios), mainly includes the SD - 208 
SB431542, SB505124 (GlaxoSmithKline) and LY - 2157 $\sim 99$ (Lilly Research Laboratories), and play a major role in the clinical trials can inhibit the growth of tumor cell metastasis proved to be a SD - 208. The SB525334 [27], LY2109761 [28] will be targeted to tumor drug resistance in some cases the drug sensitivity of tumor cells.

\section{B. TGF - beta the clinical use of the beta monoclonal antibody}

Have a highly specific monoclonal antibody that it can give full play to the role of the cleared in extracellular ligands, resulting in increased in intravenous delivery way. At present, for monoclonal antibody, had appeared on the market many never used, of course, there are applied to clinical and achieved certain results. There are three main types: single (lerdelimumab) CAT152 resistance to wood, beauty for wood single metelimumab CAT192 and husband SuoMu resistance (fresolimumab) GC - 1008.Combined with antibodies and neutralizing antibodies are similar, their molecular expression can prevent outside the cell ligand and TGF - beta receptors. Now, one of the most used in anti-tumor and tumor is the husband SuoMu list [29].

\section{V.CONCLUSION AND PROSPECT}

Pancreatic cancer is one of the high degree of malignant tumor in recent years, the incidence of a disease, early diagnosis difficult and clinical therapeutic effect is poorer, so will be able to get early diagnosis and correct operation scheme is the key to the treatment of pancreatic cancer. Especially to strengthen the screening of high-risk groups, set a reasonable treatment scheme with multi-disciplinary comprehensive treatment at the same time. On pancreatic cancer in recent years, in the process of the discussion of molecular mechanisms involved in related gene become an important direction of research, especially in pancreatic cancer and generated in the process of signal transduction of various related factors, at the same time, there have been some signaling pathways such as (TGF - beta) of the use of targeted drugs, and more or less in the clinical has achieved some success. With the development of molecular biology in recent years, gene regulation of tumor research on pancreatic cancer cells to become the same targets. Transforming growth factor for a variety of tumor cells play different important utility, although at present a lot of scholars have reached some important conclusions, but there are still many problems to be solved. TGF - resistant beta in tumor growth inhibition role at the same time, also can cause changes in the microenvironment of tumor cell. Targeted drugs against TGF - beta is vigorously developing, a lot of drugs has entered the stage of clinical trials. While at the same time of TGF - beta antitumor reaction still exist, so before TGF - beta treatment resistance is still need to do a lot of preparatory work, in order to increase therapeutic effect. Believe that through the basis of the rational experimental study and clinical trial evaluation, establish a sensitive and effective early warning and diagnostic screening system and multi-disciplinary comprehensive treatment, especially in the application of TGF - beta blockers will become an important means of treatment of pancreatic cancer and a variety of tumors and prospect.

\section{REFERENCES}

[1] Xiao-guang Ni, Ping Zhao. The research progress of invasion and metastasis of pancreatic cancer related gene. Practical journal of cancer[J].2005,20(03):97

[2] YuQingXiang. Transforming growth factor beta and tumor metastasis is reviewed. The world Chinese people digest magazine[J].2006(25):74-77

[3] High school jade, zi-hao Lin, Yuan Xiangbin. New progress of TGF - beta gene expression regulation. Foreign medical (physiology, pathology and clinical staging science) $[\mathrm{J}] .2004(03): 51-54$

[4] Jenning JC,Mohans, Linkhart TA,et al. Comparison of the biology actions of TGF beta-1 and TGF beta-2:differential activity in endotbelial cells[J].Cell Physical,1998,137(1):167-172

[5] Li Song. Transforming growth factor beta and pancreatic cancer. Huaxi medical[J].2003 (04):160-161

[6] Yong-hong Guo, Jin-yan Luo. TGF - beta superfamily and Smad signal transduction were reviewed. Medicine were reviewed[J].2005,11(08):15-18

[7] Weiser R,Wrana JI,Massague J.GS domain mutations that constitutively activate ThR-1, the downstream signaling component in the TGF beta receptor complex[J].EMBO J.1995,14(10):2199-2208

[8] GuLiJun, JieChen. TGF - beta Smads signal pathways in a super family. Foreign medical (physiological, pathological and clinical pathol science)[J].2002,22(06):49-52

[9] Attisanol,WranaJI.SignaltransductionbytheTGF-betasuperfamily[J] .Science.2002,296(5573):1646-1647

[10] Giehlk,Seidel B,Gierschik P,et al.TGF-beta repress proliferation of pancreatic carcinoma cells which correlates with smad 4-independent inhibition of ERK activation $[\mathrm{J}]$. On cogene.2000,19(39):4531-4541

[11] Liu JH, Weis, Bumette PK,et al.Function association of TGF-beta receptor II with cyclineB[J].Oncogene.1999,18(01):169-175

[12] Condeelis J,Pollard JW. Macrophages: Obligate partners for tumor Conl migration, cell invasion and metastasis[J].Cell.2006,124(02):263-266

[13] Ebert M,Fei G,Schandl L,Mawrin C,et al. Peduced PTEN expression in the pancreatic over respressing transforming growth factor-beta[J].British Journal of Cancer.2002,86(02):257-262

[14] Akhurst J,Derynck R.TGF-beta signaling in cancer- $\alpha$ double-deged sword. Trends Cell Biol.2001,(11);257-262

[15] Christin A,O'Mahony MD,et al. Transforming growth factor-betal inhibits generation of angiostatin by human pancreatic cancer cells[J].Surgey.1998,124(02):388-393

[16] THIERYJP.Epithelialmesenchymaltransitionsintumourprogression[ J].NatRewCancer.2002,2(06):442-454

[17] Volker Ellenrieder, Sandra F, WolfgangB,et al. Trasforming growth factor $\beta$, treatment leads to an epithelial-mesen chymal, tranadifferentiation of pancreatic cancer cells requiring extracellular signal-regulated kinase2 activation[J].Cancer Res.2001,61(10):4222-4228

[18] Jia-ping Zheng, Jian-min Yang. The invasion and metastasis of pancreatic cancer molecular mechanism and targeted therapy. Medical review[J].2010,16(11):41-44

[19] I takura J,Ishiwata T,Friess H,et al. Enhanced expression of vascular endothelial growth factor in human pancreatic cancer correlates with local disease progression[J].Clin Cancer Res.1997,3(08):1309-1316 
[20] Qian X,Rothman VL, Nicosia RF, et al. Expression of thrombospondin-1 in human pancreatic adenocarcinomas; role in matrix metalloproteinase-9 production[J].Pathol Oncol Res.2001,7(04):251

[21] Kasper HV,Ebert M, Molfertheiner P, et al. Expression of thrombospondin-1 in pancreatic carcinoma; correlation with microvessel density[J].Virchows Arch.2001,438(02):116

[22] Wente MN,Keane MP, Burdick MD,et al. Blockade of the chemokine receptor CXCR2 inhibits pancreatic cancer cell-induced angiogenesis[J].Cancer Letters.2006,232(02):123-130

[23] Xinyu Ren, Ahi-yong Liang, Barbara. Pancreatic cancer occurs in the process of signaling pathways. Basic medicine and clinical[J].2007,27(09):112-117

[24] Wang zhiwei,Sengupta R, Banerjee S,et al. Epidernal growth factor receptor-related protein inhibits cell growth and invasion in pancreatic cancer[J].Cancer Res.2006,66(15):7653-7660
[25] Cantley LC. The phosphoinositide3-Kinase pathway[J].Science.2002,(296):1655-1657

[26] Lin KH, Baines AT, Fiordalisi JJ,et al. Activation of ral A is critical for ras-induced tumorigenesis of human cells[J].Cancer Cells.2005,(07):533-545

[27] Kim YJ, Hwang TS, Hong YB, Bae I, Seong YS. Transforming growth factor beta receptor I inhibitor sensitizes drug-resistant pancreatic cancer cells to gemcitabine[J].Anticancer Res.2012,32(03):799-806

[28] Konpal M,Yan J,Lu X,Xu S,Lerit DA, Kang Y.Imaging transforming growth factor-beta signaling dynamics and therapeutic response in breast cancer bone metastasis[J].Nat Med.2009,15(08):960-966

[29] Trachtman H, Fervenza FC, Gipson DS, Heering P,Jayne DR, Peters H,et al.A phase 1, single-dose study of fresolimumab, an anti-TGF-Bantibody,in treatment-resistant primary focal segmental glomerulos-clerosis[J].Kidney Int.2011,79(11):1236-1243 